17.1

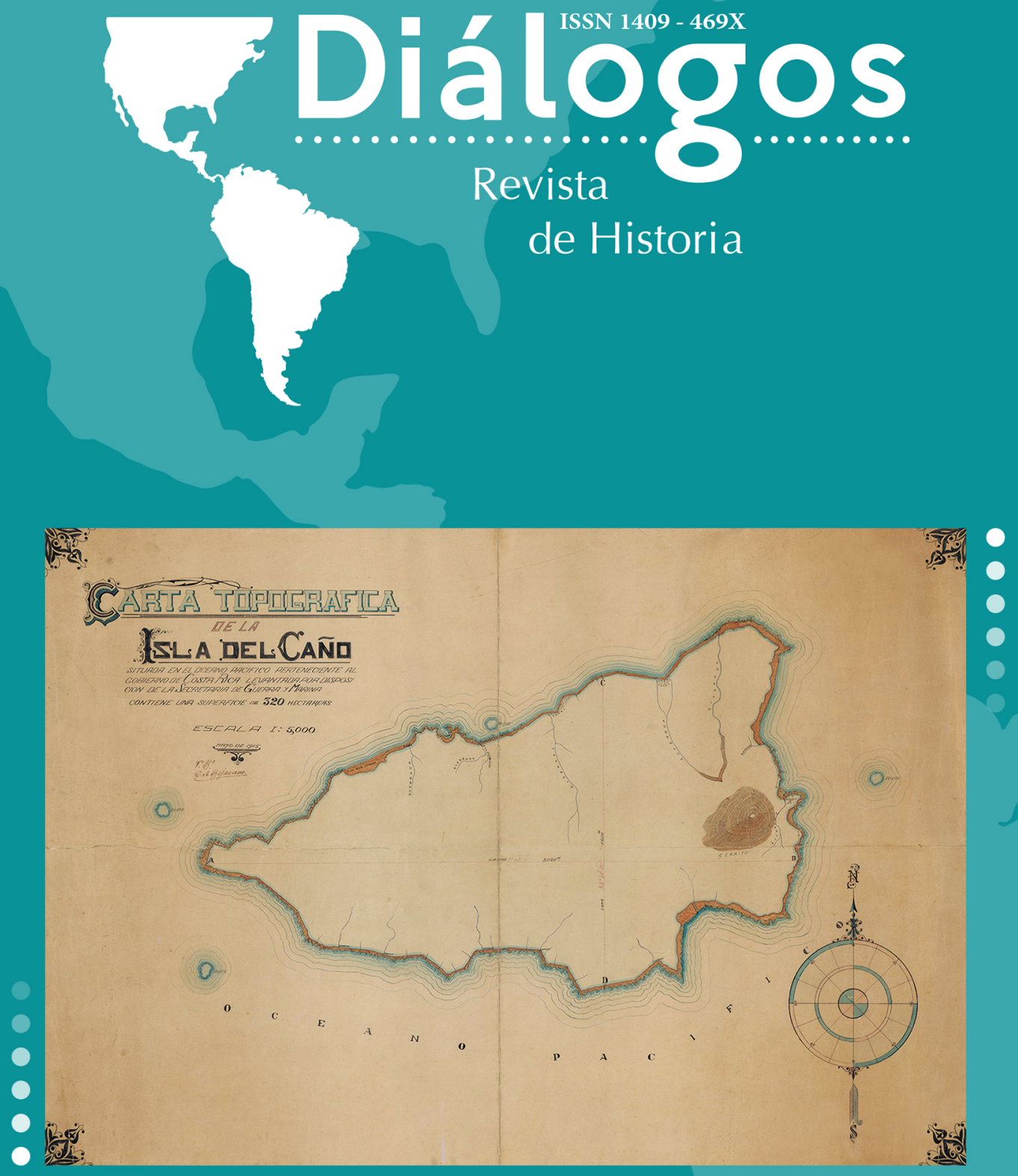

Escuela de Historia. Universidad de Costa Rica

Enero-junio 2016

url: http://revistas.ucr.ac.cr/index.php/dialogos/index 


\title{
TURISMO Y METABOLISMO SOCIAL: EFECTOS AMBIENTALES DE LA ACTIVIDAD TURÍSTICA EN LA REGIÓN ATLÁNTICO-CARIBE. 1970-2011
}

Edgar Eduardo Blanco Obando

\begin{abstract}
Resumen
Desde el enfoque del metabolismo social y el análisis de la construcción de infraestructura de servicios, durante el periodo entre 1970 y 2011, se evidencia que el desarrollo de la actividad turística en la región Atlántico-Caribe ha sido bastante modesto, uno de los más bajos del país. Esta situación provoca la existencia de reducidos daños a la naturaleza y permite a su vez, sostener que la empresa turística como unidad de apropiación mantiene un metabolismo sustentable de interacción con el medio ambiente.
\end{abstract}

Palabras claves: turismo, región Atlántico-Caribe, metabolismo social, medio ambiente, sustentabilidad.

\section{TOURISM AND SOCIAL METABOLISM: ENVIRONMENTAL EFFECTS OF TOURISM IN THE ATLANTIC-CARIBBEAN REGION. 1970-2011}

\begin{abstract}
Since the approach of social metabolism and analysis of infrastructure construction services during the period between 1970 and 2011, it appears that the development of tourism in the Atlantic-Caribbean region has been fairly modest, one of the lowest from the country. This situation causes the existence of reduced damage to nature and allows in turn, argue that the tourist business as a sustainable metabolism ownership remains with the environment of interaction.
\end{abstract}

Keywords: tourism, Atlantic-Caribbean region, social metabolism, environment, sustainability.

Fecha de recepción: 14 de enero de 2015 - Fecha de aceptación: 21 de julio de 2015

\footnotetext{
- Edgar Eduardo Blanco Obando - El autor es Doctor en Sociología por la Universitat

- Autònoma de Barcelona, se desempeña como investigador de temas ambientales y del

- desarrollo en el Centro de Investigaciones Históricas de América Central de la Universidad

- de Costa Rica y como docente en la Escuela de Lenguas Modernas de la Universidad

- de Costa Rica. Este trabajo se elaboró dentro del Programa Historia Regional/Ambiental

- Comparada del Centro de Investigaciones Históricas de América Central de la Universidad

- de Costa Rica, y contó con el apoyo de la Sede del Caribe de la Universidad de Costa Rica.
} 


\section{INTRODUCCIÓN}

La actividad turística se ha consolidado en Costa Rica como una de las más dinámicas y lucrativas, por lo que ha contado con un constante apoyo estatal y del sector privado para su crecimiento y generación de beneficios. Junto al apoyo continuo del turismo por parte de empresarios particulares, el Estado brindó un soporte más formal e importante a la actividad a partir de la década de 1970, incrementándose luego durante la década de 1990 como resultado de las reformas neoliberales que asignaron estímulos y ayudas al desarrollo del sector servicios, especialmente el turismo, al considerarse rentable y generador de divisas.

Para el año 2000, el sector servicios creó el 55,9\% de los puestos de trabajo, el mayor porcentaje del país, superando ampliamente al tradicional sector primario que generó apenas el 19,6\% de empleos (Instituto Nacional de Estadística y Censos, 2000). Desde la década de 1990, el turismo ha incrementado significativamente su aporte al producto interno bruto (PIB), al pasar del 4,6\% en 1991 al 5,8\% en 1996 y al 8,7\% en el 2001 (Instituto Costarricense de Turismo, 2005a).

Entre 2003 y 2011, las divisas por concepto de turismo se incrementaron en $\$ 776,1$ millones, al pasar de \$1 199,4 millones a \$1 975,5 millones. En comparación con otras exportaciones como el café y el banano, el turismo muestra mayor eficiencia para crear divisas; por ejemplo, en 1996 la actividad turística produjo $\$ 688,6$ millones; $\$ 303,2$ millones más que el café y $\$ 21,1$ millones más que el banano. Para 2011, el turismo creó $\$ 1$ 975,5 millones, $\$ 1$ 286,9 millones más que en 1996; además generó \$1 600,6 millones más que el café y \$1 223,1 millones más que el banano. En este mismo año, los ingresos por turismo representaron cerca de la tercera parte del total de divisas por exportaciones del país (Instituto Costarricense de Turismo, 2014).

Como actividad productiva, el turismo ocasiona impactos directos sobre el medio ambiente debido a que se apropia de recursos naturales y emplea diversos tipos de energía para su operación, lo que afecta a los ecosistemas al intensificar su extracción y excretar cantidades de desechos al final de los ciclos productivos. Es común que la empresa turística además de consumir grandes cantidades de agua y energía, se apropie de espacios naturales y los transforme en áreas cubiertas de infraestructura o en otro tipo de ecosistemas manipulados como jardines o campos de golf, y excrete desechos sólidos, líquidos y gaseosos producto de la satisfacción de las necesidades de la población visitante, que se suman a los desechos de las poblaciones locales.

En este escenario se ubica la importancia de analizar los principales efectos ambientales producidos por la actividad turística a nivel local desde la década de 1970, cuando el turismo inició su auge ascendente mediante el modelo de sol y playa con el apoyo formal del Estado y la participación de la empresa privada, hasta 2011, cuando la actividad se consolidó como una de las principales fuentes de empleo y divisas para el país. El estudio se centra en la región Atlántico-Caribe, 
que comprende los territorios de la provincia de Limón y zonas de influencia directa como los cantones de Turrialba en Cartago y Sarapiquí en Heredia; estos territorios comparten caracteres climáticos, topográficos, procesos históricos y condiciones productivas similares. La región posee recursos naturales endógenos junto a una rica diversidad étnica, elementos que han servido de sustento y estímulo para el desarrollo del turismo en la zona.

El análisis longitudinal de los efectos ambientales producidos por la actividad turística permite contextualizar una forma específica de explotación de la naturaleza junto con sus efectos sobre el medio natural, de modo que se evidencien los cambios en el tiempo sobre el medio ambiente. Para la comprensión de esta dinámica entre unidad productiva y naturaleza se ha empleado el metabolismo social, cuyo enfoque y sustento teórico aportan las herramientas para comprender desde la sustentabilidad los efectos ambientales ocasionados por una determinada actividad productiva. Entre humanos y naturaleza existe una relación dialéctica en prácticamente todos los sentidos, el metabolismo social permite el análisis y la comprensión de esta relación y sus efectos derivados.

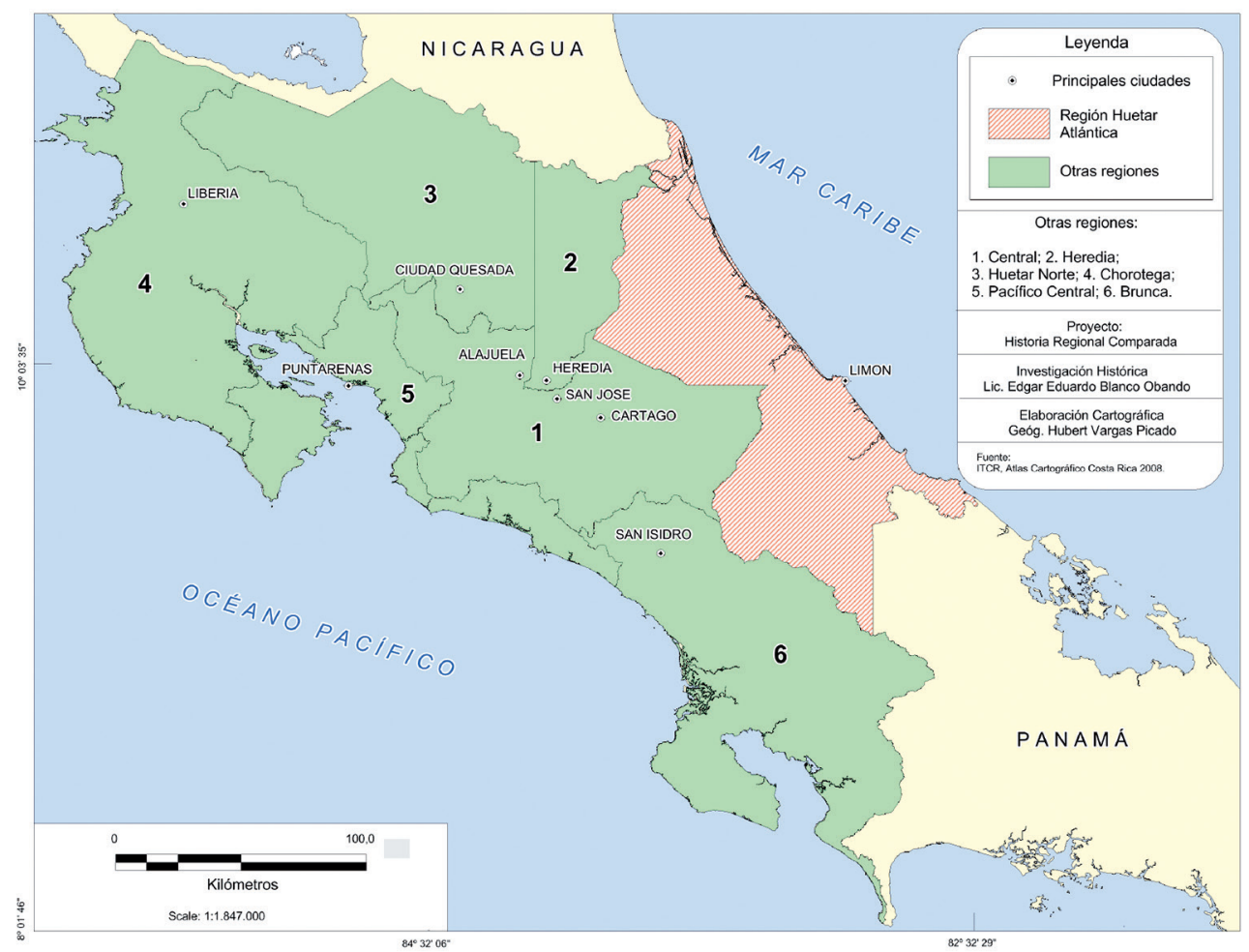

Figura 1. Mapa de la región Atlántico-Caribe de Costa Rica. Tomado de Atlas Cartográfico de Costa Rica, (p. 10), Instituto Tecnológico de Costa Rica, 2008, Costa Rica: EITCR. Derechos reservados 2008 por Edgar Eduardo Blanco Obando. Reproducido con permiso. 


\section{EL TURISMO EN COSTA RICA}

El turismo se define como el movimiento geográfico de personas por causas diversas como el ocio, la cultura, estudios, negocios, religión, salud, visitas sociales, etc. Los individuos al viajar interactúan con otras personas, hacen uso de servicios, consumen bienes y satisfacen necesidades, por lo que influyen en el dinamismo de las economías y en las relaciones sociales de las poblaciones receptoras. Este traspaso de recursos de los visitantes hacia los receptores es lo que convierte al turismo en actividad generadora de riqueza.

A partir de 1950, el turismo experimentó un auge importante con el surgimiento del denominado turismo de masas, que se consolidó gracias a circunstancias como la modernización de los medios de comunicación, el crecimiento del nivel de vida en los países desarrollados y la ausencia de conflictos bélicos de escala global (Arrieta y Rivera, 2009). Posteriormente, cambios en los gustos y necesidades de los países occidentales, consolidados como los principales demandantes de opciones turísticas, provocaron la diversificación de la oferta, por lo que el típico modelo de sol y playa pasó a interaccionar con otras propuestas urbanas y rurales como el turismo de congresos y convenciones, el turismo rural, de deporte, de aventura, el turismo cultural, de comercio, de lujo, de eventos científicos y el turismo de cruceros (Maluquer, 2011).

Para Fernández Fuster (1985), desde 1950 se dio una fase importante en la evolución del turismo moderno que promovió sus beneficios, pero no visibilizó sus consecuencias, al denominársele industria sin chimeneas y ser concebido como una actividad económica sin gran impacto sobre el medio ambiente. Esta fase se extendió hasta las últimas décadas del siglo XX, cuando surgió una fuerte crítica al turismo basada en el análisis de sus costos económicos y sociales sobre las poblaciones receptoras y la naturaleza misma. Esta crítica provocó el surgimiento del turismo sustentable como alternativa, al ocasionar, al menos en teoría, menor impacto sobre el medio ambiente y estar mejor adaptado a los requerimientos y necesidades de la sociedad del siglo XXI, la cual se autodefinió como preocupada por el cuidado y disfrute de la naturaleza y deseosa de gozar de los beneficios de un turismo de bajo impacto sobre los sectores ambiental, cultural y social de los países receptores (Arnaiz y Dachary, 2006).

En Costa Rica, el desarrollo del turismo como actividad regulada y de interés público puede ubicarse en las primeras décadas del siglo XX con la fundación de la Sociedad Anónima de Bella Vista, que tenía como objetivos atraer inmigración al país y explotar los atractivos turísticos naturales y otros servicios que se ofrecían en el Valle Central. En 1931 esta asociación privada recibió el apoyo del Estado con la fundación de la Junta Nacional de Turismo, junto a la elaboración de la Legislación Nacional de Turismo (Arrieta y Rivera, 2009), como forma de reconocer a nivel gubernamental la importancia de la actividad turística para el país. En la década de 1950, el Estado substituyó la Junta Nacional de Turismo por una institución más 
ágil y mejor financiada, que además de liderar la modernización de la industria promoviera la generación de divisas para mejorar las finanzas públicas, por lo que fundó en 1955 el Instituto Costarricense de Turismo (ICT) como institución descentralizada con carácter ministerial.

Así, el Estado se convirtió en el principal actor en la gestión del desarrollo del turismo a través del ICT, entidad que durante las décadas de 1960 y 1970 realizó una intensiva labor para transformar el turismo en una actividad moderna, regulada y lucrativa. Para los empresarios nacionales, el desarrollo del turismo debía darse acorde a las corrientes mundiales, por lo que propusieron insertar al país en las nuevas tendencias turísticas que se alejaban del tradicional turismo de masas y se acercaban a la búsqueda de experiencias más educativas, en destinos que permitieran una interacción más directa con las culturas, tradiciones y recursos naturales locales, por lo que presionaron para institucionalizar un nuevo tipo de turismo.

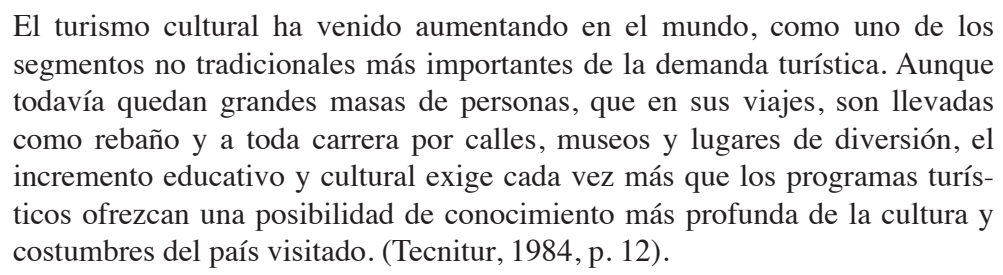

Esta visión de los empresarios turísticos impulsó la instauración del modelo del turismo ecológico, el cual además de adaptarse a las corrientes globales de preservación de la naturaleza, permite un mayor contacto con los atractivos naturales y culturales de las distintas comunidades del país:

No era así hace algunos años, cuando nuestro país intentaba atraer turistas con otros argumentos, invitándolos a conocer el "país de la amistad" y sugiriendo: "tenemos tanto que compartir".

Nuestra nación atrae hoy a los visitantes deseosos de estudiar, descubrir, aprender y disfrutar de nuestras áreas naturales, la belleza de nuestros paisajes y la diversidad de especies animales y vegetales que habitan en un territorio pequeño. Con frecuencia se habla de Costa Rica como país modelo en el desarrollo del turismo ecológico. (Budouski, 1991, p. 16).

Ese nuevo modelo también promovió entre los empresarios la adopción de prácticas respetuosas del medio natural y un mayor cuido de la naturaleza, de modo que se consolidaran el respeto y la preservación del medio ambiente como sustento de la nueva propuesta turística, con el fin de afianzar al país como destino ecoturístico mundial.

Ya no cabe construir complejos hoteleros o turísticos que no midan el impacto ambiental, cuyos diseños no se ajusten a esta nueva escala de valores. Esa influencia se dará no solamente en la construcción de complejos hoteleros, sino igualmente en la gastronomía, en el diseño de los paquetes turísticos y de los 
tours, en la aviación. Paulatinamente se irá convirtiendo en una nueva forma de vida y de apreciación del contorno en el que vivimos. (Tecnitur, 1992, p. 7).

De este modo, desde las décadas finales del siglo XX, el país ha venido consolidando su propaganda turística con base en un modelo que incorpora el cuido y disfrute de la naturaleza junto al entorno social, ofreciendo la oportunidad de disfrutar de un medioambiente conservado e inmerso en riquezas culturales autóctonas. Esta propaganda ha sido exitosa para la atracción de visitantes, cuyo arribo ha mantenido un patrón creciente; en 1996 visitaron el país 781127 turistas, monto que aumentó a 1088075 en el año 2000, a 1679051 en 2005 y a 2192059 en 2011. Así, en solo 15 años la cantidad de turistas se incrementó en 1410932 (Instituto Costarricense de Turismo, 2014).

Este significativo incremento en la llegada de viajeros se ha dado paralelamente al aumento en la oferta de servicios. Solamente en seis años, entre 2005 y 2011, las empresas con declaratoria turística en nivel de proyecto o en operación pasaron de 1355 a 1519 , siendo la mayoría las dedicadas a hospedaje, gastronomía o diversión (Instituto Costarricense de Turismo, 2014).

\section{LA ACTIVIDAD TURÍSTICA EN LA REGIÓN ATLÁNTICO-CARIBE}

El desarrollo del turismo en la región Atlántico-Caribe ha sido impulsado también por iniciativa de empresarios privados, así como por campañas y recursos del ICT. A inicios de la década de 1970 la propaganda turística era muy escasa para la región, únicamente se promocionaba Puerto Limón y la playa de Portete (Instituto Costarricense de Turismo, 1972). Durante las décadas de 1970 y 1980 se impulsó la creación de infraestructura en servicios para los turistas, como locales de hospedaje, alimentación y diversión; por lo que surgieron empresas como el hotel Acón, el motel Móvil, el hotel Kapla Inn y la discoteca La Chispa, todas ubicadas en Puerto Limón, principal polo turístico de entonces (Tecnitur, 1987).

Por su parte, la propaganda se dirigió principalmente a la promoción de los recursos culturales y naturales de la zona, como la población afrocaribeña, los ríos, playas y bosques, incorporando para su disfrute el uso turístico del ferrocarril. Se ofrecía un viaje inolvidable entre San José y Puerto Limón, debido a que el traslado en tren era garantía del contacto directo con la naturaleza, la historia caribeña, la cultura y las tradiciones locales:

Generally considered one of the great scenic train rider of the World, this $19^{\text {th }}$ century train from San José to the Caribbean port of Limon, provides an unforgettable introduction to Costa Rican life and country-side. The ten hours, 102 mile trip begins by winding its way through mountains and deep valleys, circling volcanoes and hugging cliffs, as far below the white water of the mighty Reventazon River rush by. Stops are made, some 52 in all, in small towns and villages such as Turrialba and Siquirres; food vendors board 
by dozens hawking their "empanadas", "enchiladas" and native fresh fruits. (Tecnitur, 1988, p. 11).

Para la década de 1990, la promoción turística de la región se basó en la zona del Caribe Sur, definida como poseedora de numerosas áreas protegidas y playas de gran belleza que permitían un contacto directo con la naturaleza:

Cientos de kilómetros de playa de arena blanca, compartiendo espacio con otras de arena negra y protegidas por miles de cocoteros que se levantan a través de todo el litoral caribeño, forman una región de extraordinaria belleza que se inicia prácticamente desde Limón, pasando por Cahuita, Puerto Vargas, Puerto Viejo, Cocles, Manzanillo, Punta Uva hasta Gandoca, muy cerca de las costas de Panamá. (Tecnitur, 1994, p. 18).

En la década del 2000, la oferta se dirigió a la promoción de dos polos turísticos, por un lado las llanuras húmedas del norte, colindantes con la zona de Sarapiquí, con el Parque Nacional Tortuguero y Barra del Colorado como los principales atractivos, y por otro las playas del sur, con los destinos estrella de Puerto Viejo, Gandoca-Manzanillo y el Parque Nacional Cahuita. Por su parte, Puerto Limón se convirtió en uno de los principales puertos del país para la llegada de cruceros, solo en 2005 atracaron allí 120 cruceros, el 62,5\% de los 192 navíos de este tipo que arribaron a las costas nacionales, lo que significó el desembarco de 184350 excursionistas (Instituto Costarricense de Turismo, 2005b). Sin embargo, para 2011 la llegada de cruceros se redujo al 42,5\%, al arribar solo 91 de las 214 naves que anclaron en el país en este año, por lo que la visita de este tipo de turismo se redujo considerablemente con respecto a 2005, tanto en el arribo de buques, cuyo número bajó en 22 naves, como en el desembarco de visitantes, que se redujo en 12732 excursionistas (Instituto Costarricense de Turismo, 2014).

A pesar de las acciones dirigidas al desarrollo de la actividad turística en la región, este no alcanzó durante el periodo en estudio niveles importantes, esto según la cantidad y magnitud de la infraestructura de servicios construida para satisfacer las necesidades de los visitantes. Tomando en cuenta la oferta de hospedaje para 1997, cuando el turismo ha tenido un crecimiento importante en todo el país, la provincia de Limón contaba únicamente con 2860 habitaciones, únicamente el 10,2\% del país (Instituto Costarricense de Turismo, 1998).

Para 2005, del total de empresas con declaratoria turística funcionando o en proceso de operación a nivel nacional (hospedaje, agencias de viajes, alquiler de vehículos, transporte acuático, diversión, gastronomía o líneas aéreas), solamente el 3,6\% se ubicaba en Limón, la segunda menor cantidad de estas empresas después de Cartago $(2,8 \%)$. Con respecto a las empresas de hospedaje, la provincia de Limón presentó en este mismo año, solo el 6,8\% del total nacional, segundo menor porcentaje también después de Cartago (1,2\%) (Instituto Costarricense de Turismo, 2014). 


\section{TABLA 1}

Porcentaje de empresas con declaratoria turística en proyecto o en operación y de hospedaje, por provincia en 2005

\begin{tabular}{ccc}
\hline PROVINCIA & $\begin{array}{c}\text { TOTAL DE } \\
\text { EMPRESAS }\end{array}$ & HOSPEDAJE \\
\hline San José & 48,2 & 22,5 \\
Alajuela & 11,3 & 12,9 \\
Cartago & 2,8 & 1,2 \\
Heredia & 6,6 & 7,4 \\
Guanacaste & 12,3 & 23,1 \\
Puntarenas & 15,2 & 26,1 \\
Limón & 3,6 & 6,8 \\
\hline Total & 100 & 100
\end{tabular}

También en 2005, entre las tres provincias con litoral, principal zona para el desarrollo turístico en el país, Limón presentó el menor porcentaje de empresas con declaratoria turística $(3,6 \%)$, muy por debajo de Guanacaste $(12,3 \%)$ y Puntarenas $(15,2 \%)$. Con respecto a las empresas de hospedaje, Limón también presentó el menor porcentaje $(6,8 \%)$, siendo superada ampliamente por Guanacaste $(23,1 \%)$ y Puntarenas $(26,1 \%)$ (Instituto Costarricense de Turismo, 2014).

\section{TABLA 2}

Porcentaje de habitaciones con declaratoria turística en operación, por provincia en 2005

\begin{tabular}{cc}
\hline PROVINCIA & HABITACIONES \\
\hline San José & 28,4 \\
Alajuela & 9,7 \\
Cartago & 1,0 \\
Heredia & 9,2 \\
Guanacaste & 24,2 \\
Puntarenas & 22,7 \\
Limón & 4,8 \\
\hline Total & 100
\end{tabular}


Con respecto al total de habitaciones con declaratoria turística en 2005, la provincia limonense $(4,8 \%)$ aparece también con el segundo menor porcentaje después de Cartago (1,0\%). Igual sucede en la relación con las tres provincias costeras, donde Limón se ubicó muy por debajo de Puntarenas $(22,7 \%)$ y de Guanacaste $(24,2 \%)$.

Para 2011, el patrón de crecimiento del sector turístico se mantuvo muy similar al de las décadas anteriores. Limón fue la provincia con el menor porcentaje de empresas con declaratoria turística y la segunda con menor porcentaje de empresas de hospedaje.

\section{TABLA 3}

Porcentaje del total de empresas con declaratoria turística en proyecto o en operación y de hospedaje, por provincia en 2011

\begin{tabular}{ccc}
\hline PROVINCIA & $\begin{array}{c}\text { TOTALDE } \\
\text { EMPRESAS }\end{array}$ & HOSPEDAJE \\
\hline San José & 46,8 & 23,3 \\
Alajuela & 13,7 & 15,5 \\
Cartago & 3,5 & 2,6 \\
Heredia & 8,2 & 6,1 \\
Guanacaste & 11,4 & 23,1 \\
Puntarenas & 13,4 & 24,7 \\
Limón & 3,0 & 4,7 \\
Total & 100 & 100
\end{tabular}

Nota: Adaptado de (Instituto Costarricense de Turismo, 2014).

Con respecto al porcentaje de habitaciones con declaratoria turística funcionando en Limón en 2011, este fue solo del 2,9\%, el segundo más bajo después de Cartago.

De este modo, se evidencia cómo durante el periodo en estudio sin incluir el turismo de cruceros, el crecimiento general del turismo en la región fue bajo, entre los más modestos del país a pesar de los atractivos turísticos endógenos de la zona. Con respecto a los turistas de crucero, su llegada no se refleja en la construcción de infraestructura debido a que sus necesidades se satisfacen principalmente en el barco, solo están en tierra unas horas y visitan únicamente los lugares cercanos al puerto de atraque. 
TABLA 4

Porcentaje de habitaciones con declaratoria turística en operación, por provincia en 2011

\begin{tabular}{cc}
\hline PROVINCIA & HABITACIONES \\
\hline San José & 26,5 \\
Alajuela & 12,4 \\
Cartago & 1,2 \\
Heredia & 7,6 \\
Guanacaste & 28,4 \\
Puntarenas & 21,0 \\
Limón & 2,9 \\
\hline Total & 100
\end{tabular}

Nota: Adaptado de (Instituto Costarricense de Turismo, 2014).

\section{EL METABOLISMO SOCIAL}

El metabolismo social contempla la relación mutua entre las sociedades humanas y la naturaleza en prácticamente todos los niveles, partiendo de que la evolución de los grupos humanos está en relación directa con la forma en que explotan los recursos naturales. El análisis se centra en las sociedades contextualizadas en su medio ambiente de interacción, con el concepto de sustentabilidad como uno de los principales criterios para el estudio de dicha relación dialéctica entre las acciones humanas y los ciclos de la naturaleza.

Para Toledo y González (2007), las sociedades humanas interactúan con los ecosistemas a través de un proceso metabólico determinado por la misma naturaleza humana, que lleva a explotar los recursos naturales para lograr producir y reproducir ciertas condiciones materiales de existencia. Por lo tanto, el tipo de sociedad que los humanos construyen está en función de la forma en que afecten, se apropien y transformen la naturaleza; así, el metabolismo social permite analizar estas relaciones entre humanos y medio ambiente en un contexto dinámico y dialéctico.

El metabolismo social se define como "el conjunto de procesos por medio de los cuales los humanos organizados en sociedad, independientemente de su situación en el espacio (formación social) y en el tiempo (momento histórico), se apropian, $\underline{\text { circulan, }}$ transforman, consumen y excretan materiales y/o energías provenientes del mundo natural" (Toledo y González, 2007, p. 4). Así, las comunidades humanas extraen materia y energía de los recursos naturales, que transforman para satisfacer sus necesidades, y posteriormente excretan residuos y desechos de diversos tipos de regreso al medio ambiente. 


\section{LOS PROCESOS DEL METABOLISMO SOCIAL}

El proceso metabólico inicia cuando los humanos toman materiales y energías de la naturaleza, que mediante diversos fenómenos hacen transitar, transforman y consumen. El final del proceso ocurre cuando se depositan los desechos en modo material o en emanaciones de vuelta a la naturaleza. Toledo y González (2007) definen que el proceso completo del metabolismo social se compone de cinco fenómenos bien diferenciados: apropiación (A), transformación (T), distribución (D), consumo (C) y excreción (E).

1. La apropiación representa el proceso por medio del cual los grupos humanos obtienen las materias, servicios y energías que tanto las personas como sus artefactos necesitan para mantenerse y reproducirse. La apropiación la ejecuta la llamada unidad de apropiación, que puede ser un individuo o un artefacto aislado, como una familia, una fábrica, un pueblo o una cooperativa.

2. La transformación consiste en todos los cambios realizados sobre los elementos extraídos de la naturaleza para ser consumidos o emplearse en una forma distinta a su original. Este fenómeno contempla diversas actividades como el cocimiento de los alimentos, el empleo de energías y materiales diversos para la realización de artesanías, así como el funcionamiento de fábricas y de manufacturas.

3. La distribución ocurre cuando las propias unidades de apropiación no consumen todo lo que producen ni producen todo lo que consumen, por lo que los elementos extraídos y transformados se hacen circular entre una o varias sociedades, pudiendo aumentar su volumen o las distancias que recorren antes de ser consumidos. En este proceso influyen elementos como los avances en los medios de transporte, los patrones y medios para el intercambio monetario, el desarrollo de los mercados y el mismo avance de la propiedad privada.

4. En el consumo participa la sociedad completa, incluyendo las distintas unidades de apropiación. Este proceso se determina por las diversas necesidades históricas y sociales de los humanos junto a los medios que posean para satisfacerlas, que a su vez son proporcionados por sus unidades de producción y los anteriores procesos metabólicos de transformación y distribución.

5. La excreción ocurre cuando las sociedades humanas depositan sus desechos en forma de energías y materiales de vuelta a la naturaleza, participando la sociedad completa junto con las unidades de producción. Los principales elementos corresponden a la calidad de los residuos expulsados, catalogados como amigables o no con la naturaleza, así como su cantidad. Se da especial atención a si la cantidad sobrepasa o no su capacidad de reciclaje.

Así las cosas, es en el espacio natural con la apropiación donde se inicia el metabolismo y es también el lugar donde el proceso finaliza con el depósito de los 
residuos finales. La manutención del espacio natural es esencial para asegurar la continuación del ciclo metabólico y la reproducción de la sociedad ahí inmersa, por lo que lo esencial es mantener un metabolismo ecológicamente sustentable que no comprometa la continuidad de la biomasa que aporta elementos como bosques creadores de oxígeno, espacios para el deporte o la educación, energías, combustibles, materias primas, fuentes de agua, etc. De este modo, la explotación de los ecosistemas debe darse dentro de sus propios límites para no sobrepasar sus capacidades de regeneración y no provocar bajas en las producciones agrícolas o industriales en el corto plazo, o la misma desaparición de los recursos en el largo plazo.

Según Toledo y González (2007), existen tres formas básicas de apropiación de los ecosistemas por parte de los humanos y sus unidades de producción, que son responsables de efectos sobre el medio ambiente por sus características e intensidad depredadora:

1. La apropiación sin alterar sustancialmente los ecosistemas: su estructura, dinámica, arquitectura y evolución. Aquí entran las formas de la cacería, la pesca, la recolección y el pastoreo.

2. Aquellas prácticas de apropiación que desarticulan los ecosistemas e introducen especies ya domesticadas o en proceso de domesticación. Se contemplan las formas de agricultura, de ganadería, las plantaciones forestales y la acuacultura.

3. Donde el ecosistema es conservado con fines de satisfacer intereses específicos, como la protección de especies, la obtención de servicios como el mantenimiento del clima, el abastecimiento del agua, la recreación, la educación o la investigación científica.

Según estos autores, las diferentes apropiaciones de la naturaleza causan efectos distintos en el ambiente, produciendo una categorización de este según sus efectos transformadores. Así surgen las categorías de medio ambiente natural, medio ambiente transformado o domesticado y medio ambiente conservado. Estas formas o estados de la naturaleza precisan el tipo de paisaje natural que se crea, el cual en conjunto con los espacios de hábitat de los seres humanos y con la existencia de diferentes industrias, definen los espacios donde se da el metabolismo entre las sociedades humanas y la naturaleza.

\section{EFECTOS DEL TURISMO}

La actividad turística como unidad de apropiación produce un impacto sobre el medio ambiente que está en relación con su crecimiento, consumo de energías, recursos y excreción de desechos. En general, la empresa turística se caracteriza 
por apropiarse y transformar espacios naturales, excretar cantidades importantes de desechos como gases, basura y aguas negras y alterar el paisaje natural (Calderón y Madrigal, 2002).

Debido a que la oferta turística en la región Atlántico-Caribe está compuesta por una reducida cantidad de empresas con capacidad para atender un flujo bajo de visitantes, el impacto sobre el medio ambiente es poco significativo. Por sus características, dichas empresas tienden a consumir cantidades moderadas de recursos naturales y a utilizar poca energía, por lo que no transforman el medio natural ni el uso del suelo de forma considerable, mientras que el volumen de sus excretas no suele sobrepasar los niveles de asimilación por parte de la naturaleza. Por lo tanto, el metabolismo que mantienen no altera la capacidad regenerativa de los ecosistemas y no pone en riesgo la continuidad de los recursos naturales para el disfrute de la población local presente y futura.

Este bajo impacto ambiental del turismo se evidencia al analizar uno de sus principales elementos para su operación: la construcción de infraestructura y sus efectos relacionados, como levantamiento de edificios habitacionales y comerciales, preparación de terrenos, apertura de caminos, introducción de servicios municipales, movimiento de tierras, intervención de acuíferos, talado de bosques, etc.

La construcción de infraestructura es uno de los elementos que mayor daño ambiental provoca, debido al cambio del uso del suelo que conlleva, el consumo de recursos y la excreción de desechos, esto unido al funcionamiento posterior de la empresa turística edificada, que consume cantidades de recursos y energías y excreta residuos tanto sólidos, líquidos como gaseosos durante su funcionamiento. De esta forma, es posible relacionar el incremento de la construcción debido al desarrollo de la actividad turística con un mayor impacto ambiental.

Tomando en cuenta el total del área construida en el país entre 1985 y 2006, Limón fue prácticamente la provincia donde menos se construyó; solo en 1990 y en 1995 esta provincia no presentó el menor porcentaje de área construida del país, esto debido a que en dichos años Limón recibió un importante proceso de inversión en infraestructura y viviendas dirigido a compensar los graves daños ocasionados por el terremoto de 1990 (Zapata y Blanco, 2013).

Con respecto al comportamiento de la inversión internacional inmobiliaria -uno de los sectores más poderosos e interesados en la explotación turística de las costas-, esta ha mostrado muy bajo dinamismo en la provincia limonense. En 2004 Limón recibió únicamente el 1,9\% del total invertido en el país, mientras que en 2005 el porcentaje apenas aumentó al 2,1\% (Román, 2007). Tomando en cuenta el lapso entre 2008 y 2011, la construcción en metros cuadrados en la provincia de Limón presentó en cada año los promedios más bajos del país. 
TABLA 5

Porcentajes de participación en el total de área de construcción por provincia, 1985-2006

\begin{tabular}{ccccccc}
\hline PROVINCIA & 1985 & 1990 & 1995 & 2000 & 2005 & 2006 \\
\hline San José & 51,6 & 44,7 & 33,7 & 35,5 & 33,2 & 28,7 \\
Alajuela & 13,9 & 13,7 & 22,0 & 19,8 & 17,1 & 16,3 \\
Cartago & 11,8 & 9,3 & 9,8 & 9,6 & 7,2 & 7,7 \\
Heredia & 10,0 & 13,9 & 13,8 & 14,2 & 13,8 & 14,8 \\
Guanacaste & 4,2 & 5,0 & 9,3 & 9,4 & 12,1 & 15,6 \\
Puntarenas & 4,7 & 5,9 & 5,3 & 7,3 & 12,3 & 13,5 \\
Limón & 3,8 & 7,4 & 6,1 & 4,2 & 4,3 & 3,3 \\
\hline Total & 100 & 100 & 100 & 100 & 100 & 100
\end{tabular}

Nota: Adaptado de (Román, 2007).

TABLA 6

Porcentaje de metros cuadrados de construcción registrados ante el CFIA por provincia, de 2008 a 2011

\begin{tabular}{ccccc}
\hline PROVINCIA & 2008 & 2009 & 2010 & 2011 \\
\hline San José & 28,5 & 33,3 & 26,5 & 31,7 \\
Alajuela & 17,6 & 17,6 & 16,7 & 20,4 \\
Cartago & 6,9 & 8,0 & 10,7 & 10,6 \\
Heredia & 10,0 & 11,2 & 14,5 & 11,9 \\
Guanacaste & 19,5 & 11,3 & 8,3 & 9,0 \\
Puntarenas & 12,7 & 10,8 & 17,5 & 11,0 \\
Limón & 4,8 & 7,8 & 5.8 & 5,4 \\
\hline Total & 100 & 100 & 100 & 100
\end{tabular}

Nota: Adaptado de (Colegio Federado de Ingenieros y Arquitectos, 2011). 
A pesar del escaso desarrollo del turismo y de los bajos niveles de la construcción y de las alteraciones ambientales que ocasiona, es posible identificar daños relacionados con esta unidad de apropiación. Al final de la década de 1990, se reportó vulnerabilidad y sobrecarga de los acuíferos en la Zona Norte de la región, en Barra del Colorado, Tortuguero y Parismina, áreas que concentran el mayor desarrollo turístico (Programa Estado de la Nación, 2000). En 2007, la operación de inmuebles y empresas turísticas cercanos a las costas, provocó el incremento significativo de la contaminación producto de aguas negras y desechos sólidos en Talamanca, junto a alteraciones importantes en el desove de las tortugas marinas y los ecosistemas de Tortuguero (Fonseca, 2008).

El problema de contaminación de aguas parece ser el más importante, sin embargo, debe tomarse en cuenta que el poco tratamiento que se les da a los residuos, junto con la baja calidad del servicio de alcantarillado y tratamiento de aguas negras, son responsabilidad de los gobiernos locales (Fournier y Fonseca, 2007).

Estos daños pueden considerarse leves y fácilmente asimilables por la naturaleza, por lo que no reflejan niveles importantes de consumo y excreción por parte de la unidad productiva, que no parece poseer un metabolismo insustentable que ponga en peligro la continuidad de los recursos naturales. Es posible que el elemento turístico de mayor impacto ambiental en la región sea el arribo de cruceros, debido a que transportan miles de personas que al unirse a la población local incrementan significativamente el consumo de recursos y energías, sumado a la excreción de desechos gaseosos, sólidos y líquidos; no obstante, no se cuenta con datos para cuantificar y determinar explícitamente el impacto ambiental causado.

A manera de ilustración, en la década del 2000 atracaron 801 cruceros en Puerto Limón, provocando el desembarco de 1134785 excursionistas (Fonseca, 2008), una cantidad cercana a la tercera parte de la población total de Costa Rica para ese momento. Ana Fonseca (2008), con base en datos de 1999 de la European Commission, describe el impacto que significa para la población receptora y su medio ambiente de interacción, la visita de un solo crucero:

\footnotetext{
Un típico buque para cruceros con 3 mil pasajeros y tripulación genera diariamente: 115 toneladas de aguas servidas; 960 toneladas de aguas sucias de lavanderías, duchas, lavavajillas que incluyen químicos tóxicos; 3 toneladas de agua de sentinas; 1,000 toneladas de agua de lastre que contiene flora y fauna de localidades lejanas y es descargada en puertos y bahías; 7 toneladas de basura y desechos sólidos; 60 litros de químicos tóxicos; y contaminantes del aire equivalente a 12,000 automóviles. (Fonseca, 2008, p. 31).
}

Así, a pesar de que el turismo de cruceros parece ocasionar un impacto ambiental importante, la falta de datos impide cuantificarlo en términos metabólicos y evidenciar su efecto sobre los ecosistemas y la población local en estudio. 


\section{CONCLUSIONES}

La actividad turística en la región Atlántico-Caribe presenta un bajo nivel de desarrollo, evidenciado en la reducida cantidad de empresas turísticas radicadas y la poca construcción de infraestructura de servicios; esto permite catalogar el turismo como actividad con bajo impacto ambiental en la región. Este hecho no significa que la región Atlántico-Caribe carezca de conflictos o daños ambientales, sino únicamente que el turismo como actividad productiva no produce daños significativos al ambiente debido a su escaso desarrollo.

De este modo, es posible afirmar que la actividad turística en la región analizada no presenta niveles importantes de apropiación y de excreción, capaces de atentar contra la reproducción y continuidad de los ecosistemas, por lo que mantiene un metabolismo sustentable. Por su parte, la llegada de cruceros a Puerto Limón parece producir un relevante impacto ambiental, pero este aún no ha sido cuantificado, por lo que no se conocen sus efectos sobre la reproducción y continuidad de los ecosistemas.

\section{REFERENCIAS}

Arnaiz, S. M. y Dachary, A. C. (2006). El estudio del turismo. ¿Un paradigma en formación?. Estudios y Perspectivas en Turismo, 15(2), 179-192.

Arrieta, G. y Rivera, G. (2009). El desarrollo del turismo en Guanacaste: de la Asociación Bella Vista, al Instituto Costarricense de Turismo. En J. J. Marín y R. Núñez (Comp.), Guanacaste: Historia de la (re)construcción de una región. 1850-2007 (pp. 139-156). Costa Rica: Alma Mater.

Budouski, T. (enero/febrero, 1991). Ecoturismo a la tica. Tecnitur, (31), p. 16.

Calderón, H. y Madrigal, H. (2002). Efectos de la actividad turística sobre los recursos hídricos y desechos sólidos en las zonas de estudio. En E. Fürst y W. Hein (Eds.), Turismo de larga distancia y desarrollo regional en Costa Rica (pp. 230-282). Costa Rica: DEI.

Colegio Federado de Ingenieros y Arquitectos. (2011). Indicadores CFIA de la construcción en metros cuadrados. Costa Rica: Colegio Federado de Ingenieros y Arquitectos.

Fernández, L. (1985). Introducción a la teoría y técnica del turismo. España: Alianza Editorial.

Fonseca, A. (2008). La zona marino-costera. Decimocuarto Informe Estado de la Nación en Desarrollo Humano Sostenible. Costa Rica: Proyecto Estado de la Nación.

Fournier, M. y Fonseca, A. (2007). La zona marino-costera. XIII Informe Estado de la Nación en Desarrollo Humano Sostenible. Costa Rica: Proyecto Estado de la Nación.

Instituto Costarricense de Turismo. (1972). Memoria anual 1971. Costa Rica: Instituto Costarricense de Turismo. 
Instituto Costarricense de Turismo. (1998). Memoria anual 1997. Costa Rica: Instituto Costarricense de Turismo.

Instituto Costarricense de Turismo. (2005a). Anuario estadístico de turismo. Recuperado de http:// www.visitcostarica.com/ict/paginas/statistics/Anuario_2005_especial.pdf/

Instituto Costarricense de Turismo. (2005b). Plan general de usos de la tierra y desarrollo turístico para las unidades del Caribe de Costa Rica: Caribe Norte y Caribe Sur, provincia de Limón. Costa Rica: Instituto Costarricense de Turismo.

Instituto Costarricense de Turismo. (2014). Anuarios. Recuperado de http://www.visitcostarica.com/

Instituto Nacional de Estadística y Censos. (2000). Censo de población. Costa Rica: Instituto Nacional de Estadística y Censos.

Maluquer, J. (2011). El turismo, motor fundamental de la economía de Cataluña (1951-2010). Historia Contemporánea, (42), 347-402.

Programa Estado de la Nación. (2000). VI Informe Estado de la Nación en Desarrollo Humano Sostenible. Capítulo 4: Armonía con la naturaleza. Costa Rica: Programa Estado de la Nación.

Román, M. (2007). Desarrollo turístico e inmobiliario costero y preocupaciones ambientales. Decimotercer Informe Estado de la Nación en Desarrollo Humano Sostenible. Costa Rica: Proyecto Estado de la Nación.

Tecnitur. (octubre/noviembre, 1984). Editorial. Tecnitur, p. 2.

Tecnitur. (enero/abril, 1987). Editorial. Tecnitur, p. 2.

Tecnitur. (mayo/junio, 1988). One Day Tour: Jungla Train Ride. Tecnitur, p. 11.

Tecnitur. (enero, 1992). Descubriendo el Caribe. Tecnitur, p. 7.

Tecnitur. (1994). Editorial. Tecnitur, p. 18.

Toledo, V. y González, M. (2007). El metabolismo social: Las relaciones entre la sociedad y la naturaleza. En F. Garrido, M. González, J. Serrano y J. Solana (Eds.), El paradigma ecológico en las Ciencias Sociales. España: Editorial Icaria.

Zapata, E. y Blanco, E. (2013). La región Atlántico/Caribe de Costa Rica. Las políticas de desarrollo desde el Gobierno Central y desde la región: su planteamiento inicial y los resultados finales. 1950-2011. En R. Viales (Ed.), La conformación histórica de la región Atlántico/Caribe costarricense: (Re)interpretaciones sobre su trayectoria entre el siglo XVI y el siglo XXI. Costa Rica: Editorial Nuevas Perspectivas. 